\title{
FATORES DE RISCO EM GESTANTES ATENDIDAS NO SISTEMA ÚNICO DE SAÚDE (SUS), NA REGIÃO NOROESTE DO PARANÁ
}

\author{
RISK FACTORS IN PREGNANT WOMEN ASSISTED IN UNIFIED HEALTH \\ SYSTEM (SUS), IN NORTHWEST REGION PARANÁ \\ Emilli Karine Marcomini, Ana Gabriela Fernande Frank, Nanci Virginia Kuster Paula, \\ Rosa Ikino
}

Universidade Paranaense - UNIPAR

\begin{abstract}
This is a documental, descriptive and quantitative research, whose objective is to identify the risk factors of pregnant women attended in a maternity hospital in northwestern Paraná. For data collection, the records of the hospitalization books and medical records of high-risk pregnant women admitted to the maternity ward between 2014 and 2015 were evaluated. The results evidenced the presence of 1735 pregnant women with risk factors, mostly residing in the municipality of Umuarama-PR and aged between 36 and 39 years. As for pathologies, the highest prevalence was preterm labor, followed by pregnancy-specific hypertensive disease and habitual abortion. It is considered paramount to identify these risks still in prenatal care, so that all pregnant women are stratified and assisted by a more complete care, which will provide the early detection of risks, assisting in the prevention of possible Complications.
\end{abstract}

Key words: Nursing care; Pregnancy, High-Risk; Hospitalization; The woman's health.
Resumo

Trata-se de uma pesquisa documental, descritiva, quantitativa, cujo objetivo é identificar os fatores de risco de gestantes atendidas em uma maternidade no Noroeste do Paraná. Para a coleta de dados foram avaliados os registros dos livros de internação e prontuários de gestantes de alto risco internadas na maternidade entre os anos de 2014 e 2015. Os resultados evidenciam a presença de 1735 gestantes com fatores de riscos, destas, grande parte residiam no município de Umuarama-PR e possuíam idade entre 36 a 39 anos. Quanto as patologias, a prevalência maior foi o trabalho de parto prematuro, seguido da doença hipertensiva específica da gravidez e o aborto habitual. Considera-se primordial a identificação destes riscos ainda no pré-natal, para que todas as gestantes sejam estratificadas e atendidas por uma assistência mais completa, que proporcionará a detecção precoce dos riscos, auxiliando na prevenção de possíveis complicações.

Palavras chave: Cuidados de enfermagem; Gravidez de alto risco; Hospitalização; Saúde da mulher. 


\section{Introdução}

A gestação é um fenômeno natural, em que a mulher perpassa por um período cercado de transformações, tanto corporais quanto emocionais, levando a presença de sentimentos de satisfação, felicidade, medo e angústia. A maternidade é um projeto maravilhoso na vida feminina, que se institui de dentro para fora, envolvendo muitas questões físicas ${ }^{1}$.

Apesar de ser um processo fisiológico, algumas gestantes possuem características específicas ou adquirem comorbidades que interferem no desfecho da gravidez, caracterizando assim, como gestações de alto risco ${ }^{2}$. A gestação de alto risco é conceituada pela presença de algum distúrbio ameaçador à saúde da mãe e/ou do feto, em decorrência exclusiva da gestação ou de uma alteração que já existia antes de a mulher engravidar, além disso, prejudica a evolução da gravidez, elevando os riscos de morte materna ${ }^{3}$.

O Ministério da Saúde preconiza fatores de risco gestacional como características individuais e condições sócio demográficas desfavoráveis, incluindo idade maior que 35 anos e menor que 15 anos. $^{2}$ Já o programa Rede Mãe Paraense, acrescenta alguns fatores de risco, como obesidade mórbida, cirurgia bariátrica, psicose, depressão grave, intercorrências clínicas, doenças infectocontagiosas vividas durante a gestação atual (infecção do trato urinário, doenças do trato respiratório, rubéola, toxoplasmose, etc.), Doença Hipertensiva Específica da Gravidez (DHEG) na gestação atual ${ }^{4}$. Estabelece ainda, doenças clínicas diagnosticadas pela primeira vez na gestação (cardiopatias, endocrinopatias), retardo do crescimento intrauterino, Trabalho de Parto Prematuro (TPP), placenta prévia, amniorrexe prematura (abaixo de 37 semanas), sangramento de origem uterina, isoimunização $R h$ ( $R h$ negativo), má-formação fetal confirmada e microssomia do concepto com patologias.

Assim, menciona-se que a assistência à saúde dessas gestantes que possuem fatores de risco, deve se iniciar no pré-natal, com a identificação dos riscos e consequentemente acompanhamento periódico de consultas, realização de exames, garantia de ambulatório especializado e vinculação ao hospital de referência para o momento do parto conforme o risco gestacional ${ }^{5}$.

Neste contexto, o interesse em estudar as gestações de alto risco data da década de sessenta e, como seria de se esperar, despertou a atenção de inúmeros estudiosos, no mundo inteiro, por se tratar de uma questão que envolve o nascimento e a vida de mais que um indivíduo. Além disso, a gravidez de alto risco pode contribuir para a elevação dos casos de mortalidade materna-fetal, enfatizando a relevância de mais estudos acerca do tema. Mesmo diante da redução da mortalidade materna, salienta-se que os resultados atuais ainda são considerados superiores e cada óbito ocorrido deve ser visto como uma falha no sistema de saúde ${ }^{6}$.

Tendo por base que os fatores de riscos poderem interferir no nascimento de um concepto saudável, torna-se primordial conhecêlos e identificá-los precocemente, para que se possa adotar medidas para prevenção das complicações, condizentes com a própria realidade.

Sendo assim, este trabalho teve por objetivo identificar quais foram os fatores de riscos presentes nas gestantes atendidas durante os anos de 2014 e 2015 em uma maternidade no Paraná.

\section{Metodologia}

Trata-se de uma pesquisa documental, descritiva, quantitativa, em que foram avaliados os registros dos livros de internação e prontuários de gestantes de alto risco, atendidas em uma maternidade na região Noroeste do Paraná.

A maternidade escolhida é referência em atendimento de alto risco, recebendo gestantes dos 21 municípios que compõem a 12ㅇ Regional de Saúde. Para a então pesquisa utilizou-se de apenas 11 municípios que tiveram um maior número de registros de internamentos na maternidade.

Foram incluídas todas as gestantes de alto risco, atendidas entre o período de janeiro de 2014 a dezembro de 2015. Para coleta de dados utilizou-se de um instrumento especifico elaborado por uma das autoras, contendo questões referentes aos fatores socio econômicas e as patologias que desencadeiam os riscos gestacionais. Os dados coletados foram analisados por meio de estatística descritiva de caráter quantitativo.

A pesquisa teve iniciou somente após a aprovação do Comitê de Ética em Pesquisa da Universidade Paranaense (UNIPAR) sob parecer número 1.473 .980 e transcorreu do dia 25 de abril de 2015 até 10 de junho de 2016, de segunda a sexta feira, no período das $15 \mathrm{~h} 00$ às $17 \mathrm{~h} 00$. 


\section{Resultados e discussões}

No período entre 2014 a 2015 foram realizados 1735 atendimentos a gestantes de alto risco na maternidade avaliada, sendo que 819 $(47,2 \%)$ gestantes correspondem apenas ao ano de 2014 e 916 (52,8\%) ao de 2015, denotando um aumento no percentual de mulheres atendidas.
Quanto aos municípios de residência das gestantes que compõem as 12a Regional de Saúde, observa-se que nos dois períodos estudados a maioria das gestantes de alto risco eram moradoras da cidade de Umuarama (42,83\%). Este resultado era esperado, já que dentre os municípios que abrangem a 12 응 Regional de Saúde, este é o que comporta maior número de habitantes.

Tabela 1. Município de residência das gestantes de alto risco atendidas em maternidade da região Noroeste do Paraná. Umuarama - PR, 2014-2015.

\begin{tabular}{|c|c|c|c|c|c|c|}
\hline \multirow[t]{2}{*}{ Município } & \multicolumn{2}{|c|}{2014} & \multicolumn{2}{|c|}{2015} & \multicolumn{2}{|c|}{ 2014-2015 } \\
\hline & $\mathbf{n}$ & $\%$ & $\mathbf{n}$ & $\%$ & $\mathbf{n}$ & $\%$ \\
\hline Alto Piquiri & 30 & 3,66 & 25 & 2,73 & 55 & 3,17 \\
\hline Cruzeiro do & & & & & & \\
\hline Oeste & 21 & 2,56 & 22 & 2,40 & 43 & 2,48 \\
\hline Douradina & 12 & 1,47 & 19 & 2,07 & 31 & 1,79 \\
\hline Icaraima & 31 & 3,79 & 31 & 3,38 & 62 & 3,57 \\
\hline Iporã & 20 & 2,44 & 19 & 2,07 & 39 & 2,25 \\
\hline Maria Helena & 15 & 1,83 & 20 & 2,18 & 35 & 2,02 \\
\hline Pérola & 28 & 3,42 & 30 & 3,28 & 58 & 3,34 \\
\hline Tapira & 10 & 1,22 & 20 & 2,18 & 30 & 1,73 \\
\hline Xambrê & 15 & 1,83 & 16 & 1,75 & 31 & 1,79 \\
\hline Umuarama & 343 & 41,88 & 400 & 43,67 & 743 & 42,82 \\
\hline Total & 819 & 100,00 & 916 & 100,00 & 1735 & 100,00 \\
\hline
\end{tabular}

Fonte: Hospital Maternidade / Compilação: Autor 2016.

Nos dois períodos avaliados, dentre todos os internamentos foram identificadas 475 (27,4\%) gestantes com idade considerada fator de risco (<16anos e >35 anos), sendo $54,11 \%$ destas internadas em 2014 e 45,9\% em 2015, evidenciando no período um declínio no número de internações entre os dois anos.

Tabela 2 - Faixa etária das gestantes com idade considerada de risco atendidas em maternidade da região Noroeste do Paraná. Umuarama - PR, 2014-2015.

\begin{tabular}{lcccccc}
\hline Faixa etária & Ano $\mathbf{2 0 1 4}$ & \multicolumn{3}{c}{ Ano $\mathbf{2 0 1 5}$} & \multicolumn{2}{c}{$\mathbf{2 0 1 4 - 2 0 1 5}$} \\
& $\mathrm{n}$ & $\mathbf{\%}$ & $\mathbf{n}$ & $\mathbf{\%}$ & $\mathbf{n}$ & $\mathbf{\%}$ \\
\hline $\mathbf{1 3 - 1 5}$ anos & 73 & 28,40 & 65 & 29,82 & 138 & 29,05 \\
$\mathbf{3 6 - 3 9}$ anos & 114 & 44,36 & 105 & 48,17 & 219 & 46,11 \\
$\mathbf{4 0 - 4 6}$ anos & 70 & 27,24 & 48 & 22,02 & 118 & 24,84 \\
\hline Total & $\mathbf{2 5 7}$ & $\mathbf{1 0 0}$ & $\mathbf{2 1 8}$ & $\mathbf{1 0 0}$ & $\mathbf{4 7 5}$ & $\mathbf{1 0 0}$ \\
\hline
\end{tabular}

Fonte: Hospital Maternidade / Compilação: Autor, 2016.

Os resultados demonstraram que as que apresentaram maior percentual de internações nos dois períodos estudados possuíam idade entre 36 a 39 anos $(46,11 \%)$. Ao se comparar os dois anos, observa-se que no ano de 2015 houve um discreto aumento no percentual de internações por gestantes entre 36 a 39 anos, contudo, a faixa etária ente 40 a 46 anos apresentou redução. 
Figura 1 - Percentual de internamentos de acordo com faixa etária das gestantes de alto risco (<16anos e $>35$ anos), atendidas em maternidade da região Noroeste do Paraná. Umuarama - PR, 2014-2015.

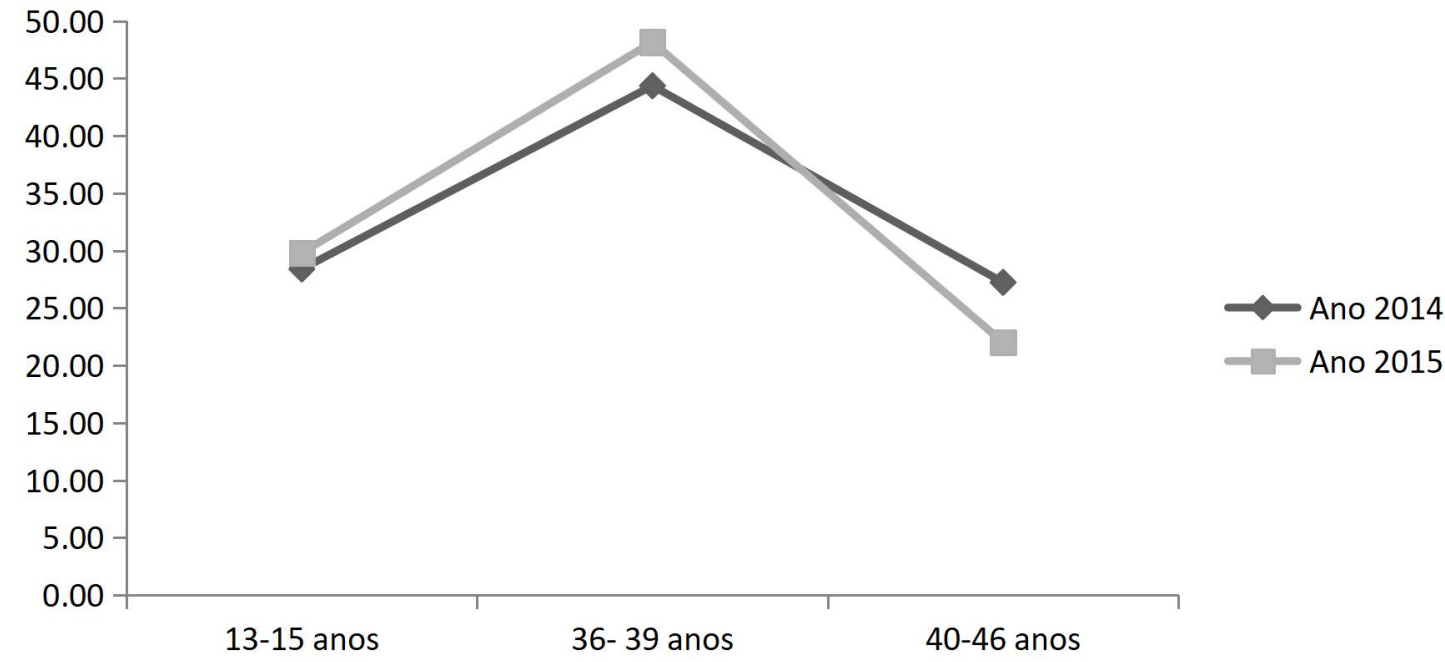

Fonte: Hospital Maternidade / Compilação: Autor, 2016.

Quanto às patologias relacionadas à gestação, a presentes nas gestantes atendidas na Tabela 3 apresenta as principais patologias maternidade.

Tabela 3 - Patologias relacionadas à gestação identificadas nas gestantes atendidas em maternidade da região Noroeste do Paraná. Umuarama - PR, 2014-2015.

\begin{tabular}{|c|c|c|c|c|c|c|}
\hline \multirow[t]{2}{*}{ Fatores de risco } & \multicolumn{2}{|c|}{2014} & \multicolumn{2}{|c|}{2015} & \multicolumn{2}{|c|}{ 2014-2015 } \\
\hline & $n$ & $\%$ & $\mathrm{Nn}$ & $\%$ & $n$ & $\%$ \\
\hline Infecção do trato urinário & 6 & 0,93 & 49 & 6,35 & 55 & 3,88 \\
\hline Trabalho de parto prematuro & 291 & 45,19 & 295 & 38,21 & 586 & 41,38 \\
\hline Oligoidrâme (abaixo de 37 semanas) & 31 & 4,81 & 15 & 1,94 & 46 & 3,25 \\
\hline Aminorrexe prematuro (abaixo de 37 semanas) & 31 & 4,81 & 36 & 4,66 & 67 & 4,73 \\
\hline Placenta previa & 1 & 0,16 & 2 & 0,26 & 3 & 0,21 \\
\hline Sangramento de origem uterina & 13 & 2,02 & 26 & 3,37 & 39 & 2,75 \\
\hline Aborto habitual & 96 & 14,91 & 156 & 20,21 & 252 & 17,80 \\
\hline Ectópicas & 7 & 1,09 & 10 & 1,30 & 17 & 1,20 \\
\hline Gemelar & 6 & 0,93 & 16 & 2,07 & 22 & 1,55 \\
\hline Hipertensão arterial (DHEG) & 160 & 24,84 & 161 & 20,85 & 321 & 22,67 \\
\hline Pré-eclâmpsia & 2 & 0,31 & 2 & 0,26 & 4 & 0,28 \\
\hline Total & 644 & 100,00 & 772 & 100,00 & 1416 & 100,00 \\
\hline
\end{tabular}

Fonte: Hospital Maternidade/ Compilação: Autor,2016.

Observou-se que o maior percentual foi evidenciado no Trabalho de parto prematuro, seguido da DHEG e o Aborto habitual, denotando aumento percentual entre os dois anos avaliados. Menciona-se também que houve uma elevação na frequência de algumas patologias durante o ano de 2015.

Além das patologias descritas na tabela, foram identificados na presente pesquisa, um caso de Infecção Sexualmente Transmissível (IST) em 2014 e quatro em 2015, somado a dois casos de incompatibilidade sanguínea (fator $\mathrm{Rh}$ ) e dois de microssomia em 2014.

A gestação é considerada de risco quando apresenta fatores que colocam a mulher próxima ao óbito ou proporciona o aparecimento de comorbidades que interferem no desfecho do ciclo gravídico puerperal.

Em relação a idade materna, gestantes menores de 16 anos ou maiores que 35 anos são 
consideradas dentro de uma faixa etária de risco, portanto, devem receber um diferencial na assistência pré-natal. Especialmente as mulheres com idade superior a 35 anos são consideradas com um risco mais elevado para desenvolver um resultado adverso ou apresentar complicações de gravidez, parto e perinatais, como a exemplo do abortamento espontâneo e gravidez ectópica7. Cabe aos serviços de saúde, um olhar atento para essas mulheres, pois atualmente a gravidez tardia tem sido cada vez mais presente na sociedade.

No que se refere as patologias gestacionais, as de maior destaque identificadas neste estudo foram o TPP e DHEG. É valido ressaltar que algumas patologias desencadeiam outras comorbidades, como é o caso do TPP que pode ser consequência de uma ITU (Infecção de Trato Urinário), amniorrexe prematura ou bolsa rota. Esse desencadeante pode estar relacionado com um grande número de intercorrências e internamentos durante o período gravídico, interferindo no nascimento de um bebê a termo ${ }^{8}$.

Apesar da ITU não apresentar percentual expressivo neste estudo, principalmente no ano de 2014, ela é uma patologia muito comum em gestantes, principalmente em decorrência das modificações anatômicas e fisiológicas que o aparelho urinário sofre durante a gravidez. Essas alterações físicas levam ao aumento do débito urinário, a estase urinária e ao esvaziamento incompleto da bexiga, provocando refluxo vesico uretral. A ITU pode ser sintomática ou assintomática e a mudança $\mathrm{PH}$ alcalino favorece o crescimento das bactérias ${ }^{9}$.

Pesquisas demonstram que bactéria coli para antígenos do Streptococcus do grupo B.1, é responsável por 70 a $80 \%$ das ITU. Dentre as gestantes com bacteriúria assintomática acompanhadas, $30 \%$ desenvolvem cistite e mais de $50 \%$ desenvolvem pielonefrite. A contaminação urinária se da através da atividade sexual, com penetração das bactérias intra-utero causando Ruptura Prematura de Membrana (RPM), provocando perda parcial ou total do líquido amniótico e acelerando consequentemente o trabalho de parto. Como resultado deste processo, pode ocorrer o abortamento antes da 20 a semana de gestação ou ainda o nascimento de um recém-nascido de baixo peso $^{8}$. Evidenciou-se que este baixo percentual de ITU encontrado no estudo, pode estar relacionando à subnotificação dos casos.

No que tange aos casos de DHEG, observouse um destaque para esta patologia, o que de fato é um fator preocupante por ser considerada como uma doença que provoca mais efeitos nocivos no organismo materno, fetal e neonatal ${ }^{4}$. Estudos epidemiológicos destacam o percentual da hipertensão em cerca de 10 a $15 \%$ das gestantes, afirmando ser um problema de saúde publica mais comum entre as grávidas, responsável por taxas elevadas de morbidade e mortalidade materna e perinatal ${ }^{10}$. A hipertensão induzida pela gravidez é uma classificação genérica entre as doenças hipertensivas durante a gestação, que incluem hipertensão gestacional (hipertensão sem proteinúria), pré-eclâmpsia (hipertensão com proteinúria) e eclâmpsia (pré-eclâmpsia com convulsões).

A eclâmpsia e a pré-eclâmpsia são complicações graves, associadas ao descontrole da pressão arterial, que podem surgir na fase final da gestação. Ela pode estar associada a gravidez em mulheres com idade maior que 40 anos ou menor que 18 anos, mulheres com história familiar de pré-eclâmpsia inclusive na família do pai, pré-eclâmpsia em uma gestação anterior, gravidez múltipla (gêmeos, trigêmeos, etc.), mulheres previamente hipertensas (hipertensão crônica) e obesas ${ }^{11}$.

Em relação a pré-eclâmpsia, evidenciou-se no estudo um percentual pequeno de casos, no entanto alguns autores destacam que é uma doença de alta incidência no Brasil, podendo chegar a $10 \%$ dos casos. Esta doença pode ocorrer devido a problemas no desenvolvimento dos vasos da placenta no início da gravidez durante a implantação da mesma no útero. Conforme a gravidez se desenvolve e a placenta cresce, a falta de uma vascularização perfeita leva a uma baixa perfusão de sangue, podendo causar isquemia placentária. A placenta, em sofrimento por falta de circulação adequada, produz uma série de substâncias as quais, ao caírem na circulação sanguínea materna, causam descontrole da pressão arterial e lesão nos rins. Os sinais mais conhecidos são o aumento da pressão arterial e a presença de proteína na urina após 20 semanas de gestação ${ }^{12}$.

Outro item de relevância, foram os casos de aborto habitual, que aumentaram entre os dois períodos avaliados. O aborto é um embrião ou feto que se exterioriza antes das 22 a 24 semanas e um abortamento é o processo que leva á exteriorização de um aborto, correspondendo normalmente à perda de uma gravidez. Cerca de 15 a $20 \%$ das gestações conhecidas, terminam em um abortamento ${ }^{13}$.

A maior parte dos abortamentos ocorre antes da gravidez atingir as 12 semanas, principalmente pelo fato do óvulo fertilizado não conseguir formar adequadamente um feto. 
Estudos remetem ao aumento do aborto relacionado a gravidez não desejada na adolescência ou a gestação na mulher com mais de 35 anos, podendo propiciar uma serie de complicações para mãe e feto, bem como alterações biológicas, psicológicas, sociais e culturais $^{14}$.

Quanto ao sangramento de origem uterina, este apresentou aumento entre os dois anos verificado, podendo estar relacionado a placenta prévia. São descritos na literatura três tipos de placenta, a prévia, a interna parcial e a total. A placenta prévia refere-se à localidade da implantação, a qual o correto seria no fundo do útero, entretanto, ela pode ser inserida parcialmente ao colo uterino ou obstruindo totalmente a área do orifício do colo uterino, onde pode ocorrer sangramento à partir da 22a semana de gestação².

Em relação a gravidez ectópica também conhecida como gestação tubária, demonstrou um aumento no número de internamentos em gestantes. Em alguns casos, a gestação ectópica ocorre na cavidade abdominal, nos ovários ou no canal cervical, impedindo deste feto crescer e se desenvolver de forma saudável. Podem ocorrer na sintomatologia clinica, a rotura das tubas uterinas, ocasionando sangramento abdominal intenso, seguido de alteração visual, desmaios e choque $^{15}$.

No que se refere a gravidez gemelar, houve um aumento significativo no número de casos, o que pode ser explicado pela incidência das técnicas de reprodução assistida. A gestação gemelar é classificada como uma de alto risco, uma vez que acarreta o parto pré-termo, proporcionando o nascimento de um bebê de baixo peso ${ }^{16}$.

Mediante as inúmeras comorbidades analisadas, verifica-se a necessidade de enquadramento das gestantes em uma unidade de saúde mais especializada, objetivando o acompanhamento das mesmas durante todo o ciclo gravídicopuerperal.

\section{Conclusão}

Diante dos dados apresentados anteriormente, conclui-se que a maioria das gestantes de risco atendidas entre os anos de 2014 e 2015 na maternidade eram moradoras do município de Umuarama. Apesar dos extremos de idade (<16 e >35 anos) serem considerados como um fator de risco nas gestantes, a predominância do estudo foi na faixa etária entre 36 a 39 anos.

Ao avaliar as patologias de risco, a prevalência significativa foi o Trabalho de Parto Prematuro, seguido da DHEG e o Aborto habitual, apresentando aumento do percentual entre os dois anos analisados. Destacando a necessidade de os profissionais de saúde possuírem conhecimento sobre a realidade estudada, identificando as comorbidades logo no início do período gestacional, para que possam ser acompanhadas durante todo o pré-natal, em decorrência dos riscos a que o binômio mãe e filho estão sujeitos.

As limitações do estudo se referem ao fato de não possuir informações relativas ao pré-natal, para relacionar a quantidade e qualidade de consultas, bem como o atendimento recebido pela gestante. Sugere-se para pesquisas futuras que contemplem a relação dos fatores de riscos com a assistência recebida, tendo em vista que um pré-natal qualificado garante o nascimento de concepto saudável, mesmo diante das patologias existentes.

Essa pesquisa buscou identificar os fatores de risco em gestantes, na percepção de que os gestores possam oferecer capacitações aos profissionais de saúde, para que estes saibam atuar frente a cada comorbidade. Conhecer a realidade de cada localidade, é um passo primordial para um atendimento efetivo e de qualidade, assim, considera-se a importância do pré-natal para o diagnostico precoce de fatores de riscos e consequentemente a vinculação desta gestante a uma estratificação de alto risco para recebimento de uma assistência de mais adequada as suas necessidades.

\section{Referências}

1. Silva LS, Pessoa FB, Pessoa DTC, Cunha VCM, Cunha CRM, Fernandes CKC. Análise das mudanças fisiológicas durante a gestação: desvendando mitos. Revista Faculdade Montes Belos (FMB) [Internet]. 2015 [citado em 08 nov 2017] 8(1):1-16. Disponível em: http://revista.fmb.edu.br/index.php/fmb/article/ viewFile/11/8

2. Brasil. Ministério da Saúde. Secretaria de Atenção à Saúde. Departamento de Ações Programáticas Estratégicas. Gestação de alto risco: manual técnico [Internet]. Brasília: 2012 [citado em 08 ago 2016]. Disponível em: http://bvsms.saude.gov.br/bvs/publicacoes/man ual tecnico gestacao alto risco.pdf

3. Luciano MP, Da Silva EF, Cecchetto FH. Orientações de enfermagem na gestação de alto risco: percepções e perfil de gestantes. Rev enferm UFPE on line [Internet]. 2011 [citado em 07 abr 2018]; 5(5):1261-266, 2011. Disponível em: 
https://periodicos.ufpe.br/revistas/revistaenfer magem/article/download/6855/6104

4. Paraná. Secretária de Estado de Saúde. Linha Guia da Rede Mãe Paranaense [Internet]. 2014 [citado em 23 fev 2018]. Disponível em: http://www.saude.pr.gov.br/arquivos/File/MaeP aranaense 2014 LinhaGuia Ed03 148×210mm 1.pdf

5. Paraná. Secretária de Estado de Saúde. Linha Guia da Rede Mãe Paranaense [Internet]. 2017 [citado em 23 fev 2018]. Disponível em: http://www.saude.pr.gov.br/arquivos/File/Linha GuiaMaeParanaense_final_2017.pdf

6. Assis TR, Viana FP, Rassi S. Estudo dos principais fatores de risco maternos nas síndromes hipertensivas da gestação. Arq Bras Cardiol [Internet]. 2008 [citado em 08 nov 2017]; 91(1):11-17. Disponível em:http://www.scielo.br/scielo.php?pid=S0066$782 \times 2008001300002 \&$ script=sci_abstract\&tlng=e $\mathrm{s}$

7. Silva JLCP, Surita FGC. Idade materna: resultados perinatais e via de parto. Rev Bras Ginecol Obstet [Internet]. 2009 [citado em 15 mar 2018].; 31(7): 321-5. Disponível em: http://www.scielo.br/pdf/rbgo/v31n7/v31n7a01. pdf

8. Figueiró-filho EA. Infecção do trato urinário na gravidez: aspectos atuais. Femina [Internet]. 2009 [citado em 06 jan 2017]; 37(3): 165-71.

Disponível

em:http://www.febrasgo.org.br/site/wpcontent/uploads/2013/05/Femina-v37n3p165.pdf

9. Figueiredo A, Gomes G, Campos A. Infecções urinárias e gravidez: diagnóstico, terapêutica e prevenção. Acta Obstet Ginecol Port [Internet]. 2012 [citado em 08 ago 2016]; 6(3):124-33. Disponível em: http://www.fspog.com/fotos/editor2/1 ficheiro 608.pdf

10. Freire CMV, Tedoldi CL. Hipertensão arterial na Gestação. Arq Bras Cardiol [Internet] 2009 [citado em 11 fev 2018]; 93(6):159-165. Disponível em: http://www.scielo.br/scielo.php?script=sci_artte $\mathrm{xt \& pid=S0066-}$

$782 \times 2009001300017 \&$ Ing=en \&nrm=iso

11. Noronha Neto C, Souza ASR, Amorim MMR. Tratamento da pré-eclâmpsia baseado em evidências. Rev Bras Ginecol Obstet [Internet]. 2010 [citado em 02 fev 2018]; 32(9): 459-68. Disponível em: http://www.scielo.br/scielo.php?script=sci_artte

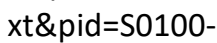

$72032010000900008 \& \operatorname{lng}=\mathrm{en} \& \mathrm{nrm}=\mathrm{iso}$
12. Cavalli RC, Sandrim VC, Santos JET, Duarte G. Predição de pré-eclâmpsia. Rev Bras Ginecol Obstet [Internet]. 2009 [citado em 06 mar 2018]; 31(1):1-4. Disponível em: http://www.scielo.br/scielo.php?script=sci_artte $\mathrm{xt} \& \mathrm{pid}=\mathrm{S0100}$

$72032009000100001 \& \operatorname{lng}=e n \& n r m=i s o$

13. Braga AS, Rios LAO, Valle NSB. Aborto "uma consequência da gravidez na adolescência". Rev Edu Meio Amb e Saúde [Internet]. 2008 [citado em 19 fev 2018]; 3(1): 76-88. Disponível em:

http://www.faculdadedofuturo.edu.br/revista/2 008/pdfs/REMAS3(1)76a88.pdf

14. Vieira EM. A questão do aborto no Brasil. Rev Bras Ginecol Obstet [Internet]. 2010 [citado em 21 abr 2018]; 32(3):103-4. Disponível em: http://www.scielo.br/pdf/rbgo/v32n3/a01v32n3. pdf

15. Elito Junior J, Montenegro NAMM, Soares RC, Camano L. Gravidez ectópica NÃO rota: diagnóstico e Tratamento. Situação Atual. Rev Bras Ginecol Obstet [Internet]. 2008 [citado em 07 set 2017]; 30(3): 149-59. Disponível em: http://www.scielo.br/scielo.php?script=sci_artte $\mathrm{xt} \& \mathrm{pid}=\mathrm{S0100}$

$72032008000300008 \&$ Ing=en \&nrm=iso

16. Nakamura PM, Cunha AA, Montenegro CA. Considerações sobre o parto na gestação gemelar diamniótica. Femina [Internet]. 2009 [citado em 08 fev 2018]; 37(8): 437-42. Disponível em: http://pesquisa.bvsalud.org/ses/resource/pt/lil$\underline{534965}$

\section{Endereço para Correspondência}

Universidade Paranaense - UNIPAR

Praça Mascarenhas de Moraes, 4282 - Zona III

CEP.: 87502-210

e-mail: emillimarcomini@hotmail.com

Recebido em 28/09/2018

Aprovado em 25/01/2018

Publicado em 13/03/2019 